\title{
Gnathostoma spinigerum
}

National Cancer Institute

\section{Source}

National Cancer Institute. Gnathostoma spinigerum. NCI Thesaurus. Code C122318.

A species of parasitic nematodes in the family Gnathostomatidae. The life cycle of G. spinigerum involves copepods of the genus Cyclops as the first intermediate host, freshwater fish as the second intermediate host, and canine and feline species, and possibly pigs, as the the definitive hosts. 